\title{
Combining Ability Analysis for Fibre Quality Traits in Desi Cotton (Gossypium arboreum L.) Across the Environments
}

\author{
V. N. Chinchane*, D. B. Deosarkar, K. S. Baig and H. V. Kalpande \\ Cotton Research Station, Mahboob Baugh Farm \\ Vasantrao Naik Marathawada Krishi Vidyapeeth, Parbhani. 431401 (M.S.), India \\ *Corresponding author
}

\begin{tabular}{|l|}
\hline Ke y w o r d s \\
Combining ability, \\
Fibre strength, \\
GCA, SCA, Upper \\
half mean length
\end{tabular}

\section{A B S T R A C T}

Cotton is one of the most important fiber and cash crop of India and plays a dominant role in the industrial and agricultural economy of the country. Fifty six crosses with fifteen parents and four checks viz., PKVDH 1,PKVSuvarna, NACH 12 and PA 255 were grown in Randomized Block Design with two replications during kharif, 2016 at three locations viz., Cotton Research Station, Mahboob Baugh Farm, Parbhani $\left(\mathrm{L}_{1}\right)$, Cotton Research Station, Nanded $\left(\mathrm{L}_{2}\right)$, and Agricultural Research Station, Badnapur $\left(\mathrm{L}_{3}\right)$.Pooled over the environments, the highest GCA effect for fibre quality traits was showed by line PA 812, PA 809andtesters CNA 449, DWDa 1402, JLA 794. Pooled over the environments, among the crosses highest SCA for fibre quality traits was reported in PA $740 \mathrm{X}$ HD 514exhibiting significant SCA for UHML (1.40), micronaire (-0.21), uniformity index (0.19) and fibre strength (2.60) pooled over the locations. Selection in further generations would be rewarding to get best transgressive segregants for fibre quality.

\section{Introduction}

Cotton, the 'white gold' enjoys a pre-eminent status among all cash crops in the country. It is grown commercially in the temperate and tropical regions of more than 70 countries. Genetic improvement in desi cotton could be gained either through selection or exploitation of specific hybrid. Therefore, more emphasis should be given to increase the seed cotton yield per unit area by developing hybrids with longer staple length with sustained yield in multiple environments.

To achieve such desirable characteristics in a new cultivar, proper breeding strategies should be followed. There is an urgent need to promote those cottons that could come closer in quality to the most sought by modern textile mills. 
Selection of parents on the basis of phenotypic performance alone is not a sound procedure, since phenotypically superior lines may yield poor recombinants in the segregating generations. It is, therefore essential that parents should be chosen on the basis of their combining ability. Combining ability analysis is the most widely used biometrical tool for identifying prospective parents and for formulating breeding procedures most likely to succeed. In heterosis breeding programme, the selection of parents or inbreds based on their morphological diversity with good combining ability is very important in producing superior hybrids. The analysis of general combining ability and specific combining ability helps in identifying potential parents or inbreds for the production of superior hybrids. The Line $\times$ Tester analysis (Kempthorne, 1957) is one of the simplest and efficient methods of evaluating large number of inbreds/parents for their combining ability. Based on the information from Line $x$ Tester analysis production of commercially viable hybrids is possible. The combining ability studies provide information on the genetic architecture of the parents as well as crosses, which is useful in developing a specific breeding strategy.

\section{Materials and Methods}

The present study comprised of seven females (lines) and eight males (testers) with four standard checks thus making $56 \mathrm{~F}_{1} \mathrm{~s}$ using Line $\mathrm{x}$ Tester mating design. These lines, testers and hybrids along with four checks were sown during kharif, 2016 at three locations viz., Cotton Research Station, Nanded (L-1), Experimental farm of Department of Agricultural Botany, VNMKV, Parbhani (L-2) and Experimental farm, Agricultural Research Station, Badnapur (L-3). The observations were recorded on days to ginning percentage, upper half mean length, micronaire value, uniformity index and fibre strength. Combining ability analysis was based on the procedure developed by Kempthorne (1957) related to design II of Comstock and Robinson (1952).

\section{Results and Discussion}

Environment wise analysis of variance for combining ability is tabulated in Table 1.The mean sum of squares due to parent vs. crosses, testers were found significant at all the locations while the variance due to lines and line $\mathrm{x}$ tester was significant at $\mathrm{E}_{1}$ only for ginning percentage. The variance due to parents was non-significant at all the locations. For upper half mean length the variances due to genotypes, parents, parent vs. crosses, crosses testers, line $\mathrm{x}$ tester were found significant at all the locations whereas lines MSS was significant at $\mathrm{E}_{2}$ and $\mathrm{E}_{3}$ only. The variances showed by genotypes, parents, parent vs. crosses, crosses, testers and line $\mathrm{x}$ testers were significant at all the locations. The variance due to lines were significant at $E_{1}$ and $E_{2}$ only for micronaire whereas for fibre strength, The variances due to genotypes, parents, parent vs. crosses, crosses, testers and line $\mathrm{x}$ tester were significant at all the locations whereas, variances due to lines were non-significant at all the locations. Environmentwise combining ability effects were tabulated in Table 2.

\section{Upper Half Mean Length (mm)}

The highest positively significant GCA among lines for improving fibre UHML was observed in PA 812 (0.80). It imparted significantly higher GCA at all the locations $\left(E_{3}=0.83, E_{2}=0.82, E_{1}=1.75\right)$. Next highest GCA for bestowing UHML was reported in PA 785 (0.53). It also exhibited relatively closer significantly higher GCA at $\mathrm{E}_{3}(0.55)$ than $E_{2}(0.53)$ and $E_{1}(0.52)$. Amongst the 
testers the tester i.e. DWDa 1402 (1.91) showed significantly positive GCA for contributing towards the progeny. It exhibited high GCA over the locations among the testers $\left(\mathrm{E}_{1}=1.93, \mathrm{E}_{2}=1.92, \mathrm{E}_{3}=1.90\right)$. The combination of this highest combiners i.e. PA 812 x DWDa $1402 \quad$ (-1.35) possessed significant negative SCA.

Amongst the crosses highest SCA was found in cross PA 832 x Digvijay (1.79). It exhibited significantly positive SCA at all the three environments $\left(\mathrm{E}_{3}=1.95, \mathrm{E}_{2}=1.79, \mathrm{E}_{1}=\right.$ 1.63). Next highest positive significant SCA was observed in PAIG $346 \times$ DWDa 1402 (1.65) $\left(\mathrm{E}_{1}=1.72, \mathrm{E}_{2} \quad 1.63, \mathrm{E}_{3}=1.60\right)$.Among the interspecific group, cross PA $832 \mathrm{x}$ Digvijay (1.79) exhibited significant and positive SCA effects. It possessed higher SCA at $E_{3}$ (1.95) than $E_{2}$ (1.79) and $E_{1}$ (1.63). This cross was followed by PA 785 x Digvijay (1.12) which exhibited high positive significant SCA at moderate yielding location $\left(\mathrm{E}_{2}=1.25\right)$ than low yielding $\left(\mathrm{E}_{3}=1.13\right)$ and high yielding environment $\left(E_{1}=0.99\right)$.

\section{Micronaire $(\mu \mathrm{g} /$ inch $)$}

The highest significant GCA in desirable direction among lines was observed in PA 812 (-0.22). It recorded highest significant GCA in desirable direction at $\mathrm{E}_{2}(-0.25)$ than $\mathrm{E}_{1}(-0.22)$ and $\mathrm{E}_{3}(-0.20)$. Among testers, highest negative significant GCA was observed in CNA 449 (-0.55). It showed highest significant GCA in desirable direction at $E_{2}(-0.57)$ than $E_{1}(-0.55)$ and $E_{3}(-0.52)$.

Among crosses, highest significant SCA in desirable direction was observed in PA $809 \mathrm{x}$ DWDa 1402 (-0.72). It showed similar performance for SCA at all the locations $\left(\mathrm{E}_{2}=\right.$ $\left.-0.75, \mathrm{E}_{3}=-0.73, \mathrm{E}_{1}=-0.70\right)$. Cross PA $812 \mathrm{x}$ AKA $8 \quad(-0.30)$ also showed very close difference over the locations $\left(\mathrm{E}_{3}=-0.38, \mathrm{E}_{1}=-\right.$ $\left.0.27, \mathrm{E}_{2}=-0.26\right)$. Out of 14 interspecific crosses, four exhibited significant and negative SCA effects. The maximum SCA effect was registered by PAIG 346 x G.Cot.23 $(-0.42) \quad\left(\mathrm{E}_{1}=-0.39, \mathrm{E}_{2}=-0.36, \mathrm{E}_{3}=-0.51\right)$ followed by PAIG 346 x Digvijay $(-0.29)$ $\left(\mathrm{E}_{1}=-0.30, \mathrm{E}_{2}=-0.32, \mathrm{E}_{3}=-0.24\right)$.

\section{Uniformity index (\%)}

Among lines highest positively significant GCA for bestowing fibre uniformity was imparted by PA 812 (0.54). It showed high positive significant GCA at $\mathrm{E}_{3}(0.57)$ than $\mathrm{E}_{1}$ $(0.55)$ and $\mathrm{E}_{2}(0.50)$. PA $785(0.44)$ was the second largest line with high GCA. It possessed high GCA at $\mathrm{E}_{1}(0.63)$ than $\mathrm{E}_{2}$ $(0.43)$ and $E_{3}(0.27)$. Among the testers, highest positive significant GCA for fibre uniformity was reported in CNA 449 (0.77). It showed highest positive significant GCA at $\mathrm{E}_{1}(0.82)$ than $\mathrm{E}_{2}(0.77)$ and $\mathrm{E}_{3}(0.72)$.

Tester DWDa 1402 showed second highest positive significant GCA (0.70) among testers. It also possessed higher GCA at $\mathrm{E}_{1}$ $(0.92)$ than $\mathrm{E}_{2}(0.68)$ and $\mathrm{E}_{3}(0.51)$.

Among crosses, highest positively significant SCA was reported in PAIG $346 \mathrm{x}$ Phule Dhanwantary (1.44). It possessed comparatively higher SCA at $\mathrm{E}_{2}$ (2.25) than $E_{3}$ (2.08) and $E_{1}$ (0.01). Next highest SCA was observed in PA $812 \times$ HD 514 (1.41) and PA $785 \times$ CNA 449 (1.37). Among the interspecific crosses, only one cross PA $832 \mathrm{x}$ G.Cot 23 (1.27) exhibited significant and positive SCA effects in pooled analysis.

\section{Fibre strength $(g /$ tex)}

Among lines highest positive significant GCA for improving fibre strength was bestowed by PA 812 (0.30). It exhibited higher GCA at $\mathrm{E}_{1}$ (0.33) than $\mathrm{E}_{3}(0.29)$ and $\mathrm{E}_{2}(0.28)$. It was accompanied by PA 785 (0.30). It also showed slightly higher GCA at $\mathrm{E}_{1}(0.34)$ than $\mathrm{E}_{3}(0.30)$ and $\mathrm{E}_{2}(0.26)$. 
Table.1 Analysis of variance of line x tester for fibre characters in different environments

\begin{tabular}{|c|c|c|c|c|c|c|c|c|c|c|c|c|c|}
\hline \multirow{3}{*}{$\begin{array}{l}\text { Source of } \\
\text { variation }\end{array}$} & \multirow[t]{3}{*}{ d.f. } & \multicolumn{12}{|c|}{ Mean sum of squares } \\
\hline & & \multicolumn{3}{|c|}{$\begin{array}{l}\text { Upper Half Mean Length } \\
\text { (mm) }\end{array}$} & \multicolumn{3}{|c|}{ Micronaire ( $\mu \mathrm{g} /$ inch) } & \multicolumn{3}{|c|}{ Uniformity index (\%) } & \multicolumn{3}{|c|}{ Fibre strength $\left(\mathrm{g} \mathrm{tex}^{-1}\right)$} \\
\hline & & $\mathbf{E}_{1}$ & $\mathbf{E}_{2}$ & $\mathbf{E}_{3}$ & $\mathbf{E}_{1}$ & $\mathbf{E}_{2}$ & $\mathbf{E}_{3}$ & $\mathbf{E}_{1}$ & $\mathbf{E}_{2}$ & $\mathbf{E}_{3}$ & $\mathbf{E}_{1}$ & $\mathbf{E}_{2}$ & $\mathbf{E}_{3}$ \\
\hline Replication & 1 & 0.81 & 0.008 & 0.19 & 0.022 & 0.033 & 0.05 & 1.00 & 0.90 & 0.14 & 0.072 & 0.70 & 0.45 \\
\hline Genotype & 70 & $7.09 * *$ & $7.41 * *$ & $7.27 * *$ & $0.45^{* *}$ & $0.45^{* *}$ & $0.44 * *$ & $3.41 * *$ & $3.55^{* *}$ & $3.24 * *$ & $6.42 * *$ & $6.46^{* *}$ & $6.59 * *$ \\
\hline Parents & 14 & $10.51 * *$ & $10.67 * *$ & $10.40 * *$ & $1.06^{* *}$ & $1.00 * *$ & $1.09 * *$ & $7.53 * *$ & $7.53 * *$ & $6.97 * *$ & $6.58 * *$ & $6.63 * *$ & $6.38 * *$ \\
\hline $\begin{array}{l}\text { Parent vs. } \\
\text { Crosses }\end{array}$ & 1 & $69.95 * *$ & $69.78 * *$ & $69.25 * *$ & $0.64 * *$ & $0.33^{* *}$ & $0.44 * *$ & 0.09 & 0.07 & 0.75 & $38.44 * *$ & $40.91 * *$ & $43.75 * *$ \\
\hline Crosses & 55 & $5.07 * *$ & $5.44 * *$ & $5.35^{* *}$ & $0.29 * *$ & $0.31 * *$ & $0.27 * *$ & $2.43 * *$ & $2.61 * *$ & $2.34 *$ & $5.80 * *$ & $5.79 * *$ & $5.97 * *$ \\
\hline Lines & 6 & 5.56 & $6.17 *$ & $6.25^{*}$ & $0.25 * *$ & $0.32 *$ & 0.24 & $3.62 *$ & 3.78 & 3.67 & 1.79 & 1.88 & 1.93 \\
\hline Testers & 7 & $20.16 * *$ & $22.48 * *$ & $22.07 * *$ & $1.31 * *$ & $1.35 * *$ & $1.13 * *$ & $8.12 * *$ & $5.22 *$ & 3.75 & $17.81 * *$ & $17.30 * *$ & $17.17 * *$ \\
\hline Line $x$ Tester & 42 & $2.49 *$ & $2.50 *$ & $2.43 * *$ & $0.12^{* *}$ & $0.14 * *$ & $0.13 * *$ & $1.31^{*}$ & $2.00 *$ & 1.92 & $4.37 * *$ & $4.43 * *$ & $4.67 * *$ \\
\hline Error & 70 & 0.43 & 0.60 & 0.51 & 0.014 & 0.012 & 0.01 & 0.78 & 1.07 & 1.50 & 0.73 & 0.54 & 0.72 \\
\hline
\end{tabular}

*,** - Significant at 5 per cent and 1 per cent level, respectively

Table 1 contd....

\begin{tabular}{|c|c|c|c|c|}
\hline \multirow[t]{3}{*}{ Source of variation } & \multirow[t]{3}{*}{ d.f. } & \multicolumn{3}{|c|}{ Mean sum of squares } \\
\hline & & \multicolumn{3}{|c|}{ Ginning percentage (\%) } \\
\hline & & $\mathbf{E}_{1}$ & $\mathbf{E}_{2}$ & $\mathbf{E}_{3}$ \\
\hline Replication & 1 & 0.03 & 0.53 & 0.50 \\
\hline Genotype & 70 & $4.05 * *$ & $4.20 * *$ & $4.46 * *$ \\
\hline Parents & 14 & 2.04 & 0.98 & 1.65 \\
\hline Parent vs. Crosses & 1 & $38.61 * *$ & $56.45 * *$ & $60.40 * *$ \\
\hline Crosses & 55 & $3.94 * *$ & $4.07 * *$ & $4.15 * *$ \\
\hline Lines & 6 & $6.38^{*}$ & 3.92 & 2.45 \\
\hline Testers & 7 & $10.61 * *$ & $13.78 * *$ & $12.92 * *$ \\
\hline Line $x$ Tester & 42 & $2.48 * *$ & 2.47 & 2.93 \\
\hline Error & 70 & 1.32 & 1.30 & 1.52 \\
\hline
\end{tabular}


Table.2 Pooled estimates of GCA effects of parents and SCA effects of crosses studied over three environments

\begin{tabular}{|c|c|c|c|c|c|c|c|c|c|c|c|c|c|}
\hline \multirow{2}{*}{$\begin{array}{l}\text { Sr. } \\
\text { No. }\end{array}$} & \multirow[t]{2}{*}{ Genotype } & \multicolumn{4}{|c|}{ Upper Half Mean Length (mm) } & \multicolumn{4}{|c|}{ Micronaire ( $\mu \mathrm{g} /$ inch) } & \multicolumn{4}{|c|}{ Uniformity index (\%) } \\
\hline & & $\mathbf{E}_{1}$ & $\mathbf{E}_{2}$ & $\mathbf{E}_{3}$ & Pooled & $\mathbf{E}_{1}$ & $\mathbf{E}_{2}$ & $\mathbf{E}_{3}$ & Pooled & $\mathbf{E}_{1}$ & $\mathbf{E}_{2}$ & $\mathbf{E}_{3}$ & Pooled \\
\hline & GCA Lines & & & & & & & & & & & & \\
\hline 1 & PA 801 & $-0.60 * *$ & $-0.61 * *$ & $-0.60 * *$ & $-0.60 * *$ & $0.04 * *$ & $0.004 *$ & $0.013 * *$ & 0.02 & $-0.70 * *$ & $-0.76 * *$ & $-0.76 * *$ & $-0.74 * *$ \\
\hline 2 & PA 740 & $-0.25 * *$ & $-0.31 * *$ & $-0.34 * *$ & $-0.30 * *$ & $0.04 * *$ & $0.05 * *$ & $0.04 * *$ & $0.05 * *$ & $-0.19 * *$ & $-0.29 * *$ & $-0.18 * *$ & -0.22 \\
\hline 3 & PA 812 & $0.75 * *$ & $0.82 * *$ & $0.83 * *$ & $0.80 * *$ & $-0.22 * *$ & $-0.25 * *$ & $-0.20 * *$ & $-0.22 * *$ & $0.55^{* *}$ & $0.50 * *$ & $0.57 *$ & $0.54 * *$ \\
\hline 4 & PA 809 & $0.40 * *$ & $0.39 * *$ & $0.39 * *$ & $0.39 * *$ & $-0.05^{* *}$ & $-0.07 * *$ & $-0.07 * *$ & $-0.06 * *$ & 0.05 & 0.08 & 0.09 & 0.08 \\
\hline 5 & PA 785 & $0.52 * *$ & $0.53 * *$ & $0.55^{* *}$ & $0.53 * *$ & $0.07 * *$ & $0.08 * *$ & $0.06 * *$ & $0.07 * *$ & $0.63 * *$ & $0.43 *$ & 0.27 & $0.44 * *$ \\
\hline 6 & PA 832 & $-0.81 * *$ & $-0.86^{* *}$ & $-0.85 * *$ & $-0.84 * *$ & $0.17 * *$ & $0.21 * *$ & $0.0^{* *}$ & $0.19 * *$ & $-0.34 * *$ & $-0.36 * *$ & $-0.41 * *$ & $-0.37 *$ \\
\hline \multirow[t]{2}{*}{7} & PAIG 346 & 0.008 & 0.05 & 0.02 & 0.02 & $-0.05 * *$ & $-0.03 * *$ & $-0.04 * *$ & $-0.04 *$ & $-0.002 * *$ & $0.42 *$ & $0.43^{*}$ & 0.28 \\
\hline & GCA Testers & & & & & & & & & & & & \\
\hline 1 & AKA 8 & $-0.71 * *$ & $-0.69 * *$ & $-0.74 * *$ & $-0.71 * *$ & $0.09 * *$ & $0.09 * *$ & $0.11 * *$ & $0.10 * *$ & 0.09 & 0.09 & $-0.006 * *$ & 0.06 \\
\hline 2 & PhuleDhanwantary & $-1.06 * *$ & $-1.05 * *$ & $-1.05 * *$ & $-1.05 * *$ & $0.39 * *$ & $0.40^{* *}$ & $0.37 * *$ & $0.38 * *$ & $-1.37 * *$ & $-0.89 * *$ & $-0.70 * *$ & $-0.99 * *$ \\
\hline 3 & CNA 449 & $1.25^{* *}$ & $1.52 * *$ & $1.43 * *$ & $1.40 * *$ & $-0.55 * *$ & $-0.57 * *$ & $-0.52 * *$ & $-0.55 * *$ & $0.82 * *$ & $0.77 * *$ & $0.72 *$ & $0.77 * *$ \\
\hline 4 & HD 514 & $-0.52 * *$ & $-0.54 * *$ & $-0.51 * *$ & $-0.52 * *$ & $0.25 * *$ & $0.25 * *$ & $0.20 * *$ & $0.24 * *$ & $-0.31 * *$ & $-0.38 * *$ & $-0.37 * *$ & $-0.35 *$ \\
\hline 5 & DWDa 1402 & $1.93 * *$ & $1.92 * *$ & $1.90 * *$ & $1.91 * *$ & $-0.27 * *$ & $-0.24 * *$ & $-0.24 * *$ & $-0.25 * *$ & $0.92 * *$ & $0.68 * *$ & $0.51 *$ & $0.70 * *$ \\
\hline 6 & JLA 794 & $1.03 * *$ & $1.00 * *$ & $1.08 * *$ & $1.03 * *$ & $-0.15 * *$ & $-0.19 * *$ & $-0.14 * *$ & $-0.16 * *$ & $0.53 * *$ & $0.50^{*}$ & $0.49 *$ & $0.50 * *$ \\
\hline 7 & Digvijay & $-0.94 * *$ & $-1.18 * *$ & $-1.14 * *$ & $-1.09 * *$ & $0.13 * *$ & $0.12 * *$ & $0.11 * *$ & $0.12 * *$ & $-0.47 * *$ & $-0.56 * *$ & $-0.41 * *$ & $-0.48 * *$ \\
\hline \multirow[t]{2}{*}{8} & G.Cot 23 & $-0.97 * *$ & $-0.98 * *$ & $-0.97 * *$ & $-0.97 * *$ & $0.12 * *$ & $0.14 * *$ & $0.11 * *$ & $0.12 * *$ & $-0.21 * *$ & $-0.19 * *$ & $-0.23 * *$ & -0.21 \\
\hline & SCA Crosses & & & & & & & & & & & & \\
\hline 1 & PA 801 x AKA 8 & 0.48 & 0.48 & 0.60 & 0.52 & $-0.18 *$ & $-0.24 * *$ & -0.12 & $-0.18 * *$ & $1.30 *$ & 1.29 & 1.06 & $1.21 * *$ \\
\hline 2 & PA 801 x PhuleDhanwantary & -0.47 & -0.45 & -0.40 & -0.46 & 0.03 & 0.07 & 0.13 & 0.08 & -0.30 & -0.67 & -1.14 & -0.70 \\
\hline 3 & PA 801 x CNA 449 & 0.02 & -0.35 & -0.30 & -0.20 & -0.02 & 0.02 & -0.02 & -0.009 & -0.48 & -0.54 & -0.32 & -0.44 \\
\hline 4 & PA 801 x HD 514 & $-1.09 *$ & -1.03 & -0.93 & $-1.02 * *$ & $0.23 * *$ & 0.15 & 0.14 & $0.17 * *$ & -0.28 & -0.20 & -0.04 & -0.17 \\
\hline 5 & PA 801 x DWDa 1402 & 0.85 & 0.79 & 0.99 & $0.87 * *$ & $-0.19 *$ & $-0.19 *$ & $-0.19 *$ & $-0.192 * *$ & 0.52 & 0.80 & 1.03 & 0.78 \\
\hline 6 & PA 801 x JLA 794 & -0.45 & -0.39 & -0.56 & -0.46 & 0.09 & 0.04 & 0.09 & 0.07 & -0.16 & -0.12 & -0.06 & -0.11 \\
\hline 7 & PA 801 x Digvijay & 0.22 & 0.40 & 0.32 & 0.31 & -0.08 & 0.01 & -0.03 & -0.03 & -0.17 & -0.08 & -0.19 & -0.14 \\
\hline 8 & PA 801 x G.Cot 23 & 0.45 & 0.55 & 0.35 & 0.45 & 0.12 & 0.15 & -0.01 & 0.09 & -0.44 & -0.47 & -0.33 & -0.41 \\
\hline 9 & PA $740 \times$ AKA 8 & 0.38 & 0.37 & 0.35 & 0.36 & 0.04 & 0.08 & 0.07 & 0.06 & 0.80 & 0.91 & 0.67 & 0.79 \\
\hline 10 & PA 740 x PhuleDhanwantary & $-1.42 *$ & $-1.31 *$ & $-1.37 * *$ & $-1.36 * *$ & -0.06 & -0.04 & -0.04 & -0.05 & -0.80 & -1.30 & -1.39 & $-1.16 * *$ \\
\hline
\end{tabular}


Table.2 (Contd...)

\begin{tabular}{|c|c|c|c|c|c|c|c|c|c|c|c|c|c|}
\hline \multirow{2}{*}{$\begin{array}{l}\text { Sr. } \\
\text { No. }\end{array}$} & \multirow[t]{2}{*}{ Genotype } & \multicolumn{4}{|c|}{ Upper Half Mean Length (mm) } & \multicolumn{4}{|c|}{ Micronaire $(\mu \mathrm{g} /$ inch $)$} & \multicolumn{4}{|c|}{ Uniformity index (\%) } \\
\hline & & $\mathbf{E}_{1}$ & $\mathbf{E}_{2}$ & $\mathbf{E}_{3}$ & Pooled & $\mathbf{E}_{1}$ & $\mathbf{E}_{2}$ & $\mathbf{E}_{3}$ & Pooled & $\mathbf{E}_{1}$ & $\mathbf{E}_{2}$ & $\mathbf{E}_{3}$ & Pooled \\
\hline 11 & PA $740 \times$ CNA 449 & 0.22 & 0.09 & 0.08 & 0.13 & $-0.22 *$ & $-0.23 * *$ & $-0.25 * *$ & $-0.23 * *$ & -0.88 & -0.86 & -0.74 & -0.83 \\
\hline 12 & PA $740 \times$ HD 514 & $1.35^{* *}$ & $1.48 * *$ & $1.37 * *$ & $1.40 * *$ & $-0.23 * *$ & $-0.24 * *$ & $-0.17 *$ & $-0.21 * *$ & 0.30 & 0.18 & 0.09 & 0.19 \\
\hline 13 & PA $740 \times$ DWDa 1402 & $-1.40 * *$ & $-1.41 *$ & $-1.17 *$ & $-1.32 * *$ & $0.19 *$ & 0.15 & $0.16^{*}$ & $0.16^{* *}$ & 0.83 & 1.33 & 1.37 & $1.17 * *$ \\
\hline 14 & PA 740 x JLA 794 & 0.19 & 0.25 & 0.24 & 0.22 & -0.02 & 0.01 & -0.08 & -0.03 & 0.31 & 0.50 & 0.45 & 0.42 \\
\hline 15 & PA 740 x Digvijay & 0.38 & 0.20 & 0.14 & 0.24 & 0.01 & -0.07 & -0.007 & -0.02 & 0.41 & 0.04 & 0.38 & 0.27 \\
\hline 16 & PA 740 x G.Cot 23 & 0.30 & 0.33 & 0.34 & 0.32 & $0.29 * *$ & $0.33^{* *}$ & $0.32 * *$ & $0.31 * *$ & -0.97 & -0.80 & -0.84 & -0.86 \\
\hline 17 & PA 812 x AKA 8 & 0.33 & 0.36 & 0.20 & 0.29 & $-0.27 * *$ & $-0.26 * *$ & $-0.38 * *$ & $-0.30 * *$ & -1.01 & -1.16 & -1.05 & $-1.07 *$ \\
\hline 18 & PA 812 x PhuleDhanwantary & 0.58 & 0.55 & 0.63 & 0.58 & -0.09 & $-0.19 *$ & -0.14 & $-0.14 * *$ & $-1.58 *$ & $-1.92 *$ & -0.66 & $-1.38 * *$ \\
\hline 19 & PA 812 x CNA 449 & -0.69 & 0.47 & 0.47 & 0.083 & 0.15 & $0.21 * *$ & $0.15^{*}$ & $0.17 * *$ & -0.61 & -0.55 & -0.57 & -0.57 \\
\hline 20 & PA 812 x HD 514 & $0.94 *$ & 0.94 & 0.76 & $0.88 * *$ & -0.03 & 0.001 & 0.04 & 0.00 & $1.46^{*}$ & $1.57^{*}$ & 1.21 & $1.41 * *$ \\
\hline 21 & PA 812 x DWDa 1402 & $-1.31 * *$ & $-1.35^{*}$ & $-1.42 * *$ & $-1.35^{* *}$ & -0.03 & -0.009 & -0.02 & -0.02 & 0.16 & 0.33 & 0.17 & 0.22 \\
\hline 22 & PA 812 x JLA 794 & -0.81 & -0.99 & -0.79 & $-0.86^{* *}$ & $0.41 * *$ & $0.35 * *$ & $0.35^{* *}$ & $0.37 * *$ & -0.44 & -0.37 & -0.63 & -0.47 \\
\hline 23 & PA 812 x Digvijay & 0.36 & -0.56 & -0.41 & -0.20 & 0.04 & 0.01 & 0.09 & 0.05 & 0.64 & 0.70 & 0.46 & 0.59 \\
\hline 24 & PA 812 x G.Cot 23 & 0.59 & 0.58 & 0.57 & 0.58 & -0.16 & -0.11 & -0.08 & $-0.11 * *$ & $1.37 *$ & 1.39 & 1.07 & $1.27 * *$ \\
\hline 25 & PA 809 x AKA 8 & $-1.02 *$ & $-1.10 *$ & $-1.06^{*}$ & $-1.05 * *$ & 0.14 & $0.20 *$ & 0.11 & $0.15^{* *}$ & -0.51 & -0.38 & -0.30 & -0.39 \\
\hline 26 & PA 809 x PhuleDhanwantary & $1.23 *$ & $1.16^{*}$ & $1.27 *$ & $1.22 * *$ & 0.15 & $0.23 * *$ & $0.20 * *$ & $0.19 * *$ & 0.95 & 0.50 & 0.41 & 0.62 \\
\hline 27 & PA 809 x CNA 449 & -0.18 & -0.35 & -0.41 & -0.31 & $-0.22 *$ & $-0.33 * *$ & $-0.23 * *$ & $-0.26 * *$ & 0.81 & 0.91 & 0.69 & 0.80 \\
\hline 28 & PA 809 x HD 514 & -0.40 & -0.43 & -0.35 & -0.39 & $0.27 * *$ & $0.29 * *$ & $0.25^{* *}$ & $0.26^{* *}$ & -1.12 & -1.06 & -0.92 & $-1.03 *$ \\
\hline 29 & PA 809 x DWDa 1402 & $1.45^{* *}$ & $1.39 *$ & $1.29 *$ & $1.37 * *$ & $-0.70 * *$ & $-0.75 * *$ & $-0.73 * *$ & $-0.72 * *$ & 0.59 & 0.87 & 1.15 & 0.87 \\
\hline 30 & PA 809 x JLA 794 & $1.25^{* *}$ & $1.36^{*}$ & $1.37 * *$ & $1.32 * *$ & -0.12 & -0.15 & $-0.17 *$ & $-0.14 * *$ & 0.26 & 0.23 & 0.18 & 0.22 \\
\hline 31 & PA 809 x Digvijay & $-2.28 * *$ & $-2.06 * *$ & $-1.97 * *$ & $-2.10 * *$ & 0.10 & 0.09 & 0.05 & 0.08 & -0.85 & -0.82 & -0.89 & -0.85 \\
\hline 32 & PA 809 x G.Cot 23 & -0.05 & 0.04 & -0.15 & -0.053 & $0.39 * *$ & $0.41 * *$ & $0.51 * *$ & $0.44 * *$ & -0.13 & -0.25 & -0.31 & -0.23 \\
\hline 33 & PA $785 \times$ AKA 8 & $-0.94 *$ & -0.93 & -0.83 & $-0.90 * *$ & -0.08 & -0.01 & -0.07 & -0.09 & -0.07 & 0.18 & 0.41 & 0.17 \\
\hline 34 & PA $785 \times$ PhuleDhanwantary & -0.59 & -0.55 & -0.65 & -0.59 & $-0.31 * *$ & $-0.30 * *$ & $-0.31 * *$ & $-0.30 * *$ & 0.36 & 0.20 & -0.19 & 0.12 \\
\hline 35 & PA 785 x CNA 449 & $1.29 * *$ & 1.07 & $1.20 *$ & $1.18 * *$ & $0.37 * *$ & $0.37 * *$ & $0.28 * *$ & $0.33 * *$ & 1.13 & $1.51 *$ & 1.46 & $1.37 * *$ \\
\hline 36 & PA $785 \times$ HD 514 & -0.22 & -0.32 & -0.40 & -0.31 & -0.05 & -0.02 & -0.12 & -0.07 & 0.36 & 0.54 & 0.79 & 0.56 \\
\hline 37 & PA 785 x DWDa 1402 & -0.48 & -0.42 & -0.51 & -0.46 & $0.38 * *$ & $0.41 * *$ & $0.42 * *$ & $0.40 * *$ & -0.97 & $-2.38 * *$ & $-3.09 * *$ & $-2.14 * *$ \\
\hline 38 & PA 785 x JLA 794 & -0.68 & -0.75 & -0.65 & $-0.69 *$ & -0.05 & -0.02 & 0.03 & -0.01 & 0.45 & 0.82 & 1.01 & 0.76 \\
\hline 39 & PA 785 x Digvijay & $0.99 *$ & $1.25 *$ & $1.13^{*}$ & $1.12 * *$ & -0.03 & 0.006 & -0.10 & -0.04 & -0.49 & -0.32 & -0.21 & -0.34 \\
\hline 40 & PA 785 x G.Cot 23 & 0.62 & 0.65 & 0.71 & $0.66^{*}$ & $-0.22 *$ & $-0.34 * *$ & -0.11 & $-0.22 * *$ & -0.77 & -0.56 & -0.20 & -0.50 \\
\hline
\end{tabular}


Table.2 (Contd...)

\begin{tabular}{|c|c|c|c|c|c|c|c|c|c|c|c|c|c|}
\hline \multirow{2}{*}{$\begin{array}{l}\text { Sr. } \\
\text { No. }\end{array}$} & \multirow[t]{2}{*}{ Genotype } & \multicolumn{4}{|c|}{ Upper Half Mean Length (mm) } & \multicolumn{4}{|c|}{ Micronaire ( $\mu \mathrm{g} / \mathrm{inch})$} & \multicolumn{4}{|c|}{ Uniformity index $(\%)$} \\
\hline & & $\mathbf{E}_{1}$ & $\mathbf{E}_{2}$ & $\mathbf{E}_{3}$ & Pooled & $\mathbf{E}_{1}$ & $\mathbf{E}_{2}$ & $\mathbf{E}_{3}$ & Pooled & $\mathbf{E}_{1}$ & $\mathbf{E}_{2}$ & $\mathbf{E}_{3}$ & Pooled \\
\hline 41 & PA $832 \times$ AKA 8 & -0.20 & -0.15 & -0.20 & -0.18 & 0.02 & -0.002 & 0.02 & 0.014 & -0.09 & -0.03 & -0.14 & -0.08 \\
\hline 42 & PA 832 x PhuleDhanwantary & $0.94 *$ & 0.81 & 0.92 & $0.89 * *$ & 0.04 & 0.008 & 0.03 & 0.027 & $1.36^{*}$ & 0.94 & 0.88 & $1.06^{*}$ \\
\hline 43 & PA 832 x CNA 449 & $-1.57 *$ & $-1.70 * *$ & $-1.72 * *$ & $-1.66 * *$ & -0.13 & -0.14 & -0.14 & $-0.13 * *$ & 0.23 & 0.02 & 0.26 & 0.17 \\
\hline 44 & PA $832 \times$ HD 514 & 0.32 & 0.28 & 0.32 & 0.30 & -0.16 & $-0.16^{*}$ & -0.001 & $-0.10 *$ & -0.73 & -0.60 & -0.59 & -0.64 \\
\hline 45 & PA 832 x DWDa 1402 & -0.74 & -0.72 & -0.78 & $-0.74 *$ & 0.16 & $0.23 * *$ & 0.09 & $0.16^{* *}$ & -0.85 & -0.59 & -0.43 & -0.62 \\
\hline 46 & PA 832 x JLA 794 & -0.84 & -0.71 & -0.97 & $-0.83^{*}$ & $-0.19 *$ & -0.13 & -0.10 & $-0.13 * *$ & -0.46 & -0.58 & -0.64 & -0.56 \\
\hline 47 & PA 832 x Digvijay & $1.63 * *$ & $1.79 * *$ & $1.95 * *$ & $1.79 * *$ & $0.26 * *$ & $0.27 * *$ & $0.23 * *$ & $0.25 * *$ & 0.44 & 0.62 & 0.55 & 0.53 \\
\hline 48 & PA 832 x G.Cot 23 & 0.46 & 0.39 & 0.48 & 0.44 & -0.02 & -0.07 & -0.13 & -0.07 & 0.09 & 0.23 & 0.11 & 0.14 \\
\hline 49 & PAIG 346 x AKA 8 & $0.97 *$ & 0.98 & 0.94 & $0.96 * *$ & $0.34 * *$ & $0.32 * *$ & $0.38 * *$ & $0.34 * *$ & -0.42 & -0.80 & -0.65 & -0.62 \\
\hline 50 & PAIG 346 xPhuleDhanwantary & -0.28 & -0.21 & -0.32 & -0.26 & $0.25 * *$ & $0.21 *$ & 0.13 & $0.19 * *$ & 0.01 & $2.25^{* *}$ & $2.08^{*}$ & $1.44 * *$ \\
\hline 51 & PAIG 346 x CNA 449 & 0.91 & 0.77 & 0.66 & $0.78 *$ & 0.07 & 0.11 & $0.21 * *$ & $0.13 * *$ & -0.21 & -0.49 & -0.78 & -0.49 \\
\hline 52 & PAIG 346 x HD 514 & -0.91 & -0.92 & -0.77 & $-0.86 * *$ & -0.03 & -0.02 & -0.13 & -0.05 & 0.002 & -0.43 & -0.55 & -0.32 \\
\hline 53 & PAIG 346 x DWDa 1402 & $1.63 * *$ & $1.72 * *$ & $1.60 * *$ & $1.65 * *$ & $0.19 *$ & 0.16 & $0.28 * *$ & $0.21 * *$ & -0.29 & -0.37 & -0.20 & -0.28 \\
\hline 54 & PAIG 346 x JLA 794 & $1.33 * *$ & $1.23 *$ & $1.36^{* *}$ & $1.30 * *$ & -0.12 & -0.10 & -0.12 & $-0.11 *$ & 0.04 & -0.48 & -0.31 & -0.25 \\
\hline 55 & PAIG 346 x Digvijay & $-1.29 * *$ & -1.02 & $-1.17 *$ & $-1.16^{* *}$ & $-0.30 * *$ & $-0.32 * *$ & $-0.24 * *$ & $-0.29 * *$ & 0.03 & -0.14 & -0.10 & -0.07 \\
\hline \multirow[t]{5}{*}{56} & PAIG 346 x G.Cot 23 & $-2.36 * *$ & $-2.55 * *$ & $-2.31 * *$ & $-2.40 * *$ & $-0.39 * *$ & $-0.36 * *$ & $-0.51 * *$ & $-0.42 * *$ & 0.84 & 0.46 & 0.50 & 0.60 \\
\hline & S.E. \pm & & & & & & & & & & & & \\
\hline & Lines & 0.164 & 0.193 & 0.179 & 0.11 & 0.029 & 0.027 & 0.026 & 0.016 & 0.221 & 0.259 & 0.306 & 0.16 \\
\hline & Tester & 0.176 & 0.206 & 0.191 & 0.12 & 0.031 & 0.029 & 0.028 & 0.017 & 0.237 & 0.277 & 0.327 & 0.17 \\
\hline & Crosses & 0.465 & 0.546 & 0.507 & 0.32 & 0.084 & 0.078 & 0.074 & 0.045 & 0.627 & 0.732 & 0.866 & 0.45 \\
\hline
\end{tabular}

*,** - Significant at 5 per cent and 1 per cent level, respectively 
Table.2 (Contd...)

\begin{tabular}{|c|c|c|c|c|c|c|c|c|c|}
\hline \multirow{2}{*}{$\begin{array}{l}\text { Sr. } \\
\text { No. }\end{array}$} & \multirow[t]{2}{*}{ Genotype } & \multicolumn{4}{|c|}{ Fibre strength (g/tex) } & \multicolumn{4}{|c|}{ Ginning percentage $(\%)$} \\
\hline & & $\mathbf{E}_{1}$ & $\mathbf{E}_{2}$ & $\mathbf{E}_{3}$ & Pooled & $\mathbf{E}_{1}$ & $\mathbf{E}_{2}$ & $\mathbf{E}_{3}$ & Pooled \\
\hline & GCA Lines & & & & & & & & \\
\hline 1 & PA 801 & $-0.04 *$ & 0.10 & 0.04 & 0.03 & $-0.06 * *$ & -0.22 & $-0.64^{*}$ & -0.31 \\
\hline 2 & PA 740 & 0.09 & 0.03 & 0.07 & 0.07 & $0.60 * *$ & 0.57 & 0.13 & $0.43 * *$ \\
\hline 3 & PA 812 & $0.33 * *$ & $0.28 * *$ & $0.29 *$ & $0.30 *$ & $-0.62 * *$ & -0.35 & -0.25 & $-0.40 * *$ \\
\hline 4 & PA 809 & $-0.34 * *$ & $-0.36 * *$ & $-0.28 * *$ & $-0.32 * *$ & $0.76^{* *}$ & 0.54 & 0.36 & $0.55 * *$ \\
\hline 5 & PA 785 & $0.34 * *$ & $0.26 * *$ & $0.30 *$ & $0.30 * *$ & $-0.43 * *$ & -0.07 & 0.23 & -0.09 \\
\hline 6 & PA 832 & $-0.54 * *$ & $-0.58 * *$ & $-0.64 * *$ & $-0.59 * *$ & $-0.77 * *$ & $-0.76^{* *}$ & -0.26 & $-0.60 * *$ \\
\hline \multirow[t]{2}{*}{7} & PAIG 346 & 0.17 & $0.28 * *$ & $0.21 *$ & 0.22 & $0.53 *$ & 0.29 & 0.43 & $0.42 *$ \\
\hline & GCA Testers & & & & & & & & \\
\hline 1 & AKA 8 & $-0.22 * *$ & $-0.23 * *$ & $-0.22 * *$ & -0.22 & $0.80 * *$ & $0.99 * *$ & $1.22 * *$ & $1.00 * *$ \\
\hline 2 & PhuleDhanwantary & -0.99 & $-1.00 * *$ & $-1.06 * *$ & $-1.01 * *$ & $-0.28 * *$ & 0.10 & 0.25 & 0.02 \\
\hline 3 & CNA 449 & $0.99 * *$ & $0.99 * *$ & $0.96 * *$ & $0.98 * *$ & $-0.31 * *$ & $-0.69 *$ & -0.56 & $-0.52 * *$ \\
\hline 4 & HD 514 & $-1.06 * *$ & $-1.03 * *$ & $-0.95 * *$ & $-1.01 * *$ & $1.77 * *$ & $1.74 * *$ & $1.50 * *$ & $1.67 * *$ \\
\hline 5 & DWDa 1402 & $1.93 * *$ & $1.93 * *$ & $1.92 * *$ & $1.92 * *$ & $-0.91 * *$ & $-1.16^{* *}$ & $-1.12 * *$ & $-1.06 * *$ \\
\hline 6 & JLA 794 & $0.88 * *$ & $0.83 * *$ & $0.84 * *$ & $0.85^{* *}$ & $-0.28 * *$ & -0.28 & -0.65 & $-0.40 *$ \\
\hline 7 & Digvijay & $-0.96^{*}$ & $-0.89 * *$ & $-0.91 * *$ & $-0.92 * *$ & $-0.66 * *$ & $-0.96 * *$ & $-0.80 *$ & $-0.81 * *$ \\
\hline \multirow[t]{2}{*}{8} & G.Cot 23 & $-0.57 *$ & $-0.59 * *$ & $-0.59 * *$ & $-0.58 * *$ & $-0.13 * *$ & 0.26 & 0.16 & 0.09 \\
\hline & SCA Crosses & & & & & & & & \\
\hline 1 & PA 801 x AKA 8 & 0.61 & 0.36 & 0.28 & 0.41 & 0.57 & 0.056 & 0.21 & 0.27 \\
\hline 2 & PA 801 x PhuleDhanwantary & $-2.42 *$ & -2.30 & $-2.33 * *$ & $-2.35^{* *}$ & -0.23 & -0.19 & 0.10 & 0.19 \\
\hline 3 & PA 801 x CNA 449 & $1.29 *$ & $1.26^{*}$ & $1.40 *$ & $1.31 * *$ & 1.07 & 0.63 & -0.07 & 0.54 \\
\hline 4 & PA $801 \times$ HD 514 & -0.65 & -0.94 & -1.03 & $-0.87 *$ & -0.56 & -0.76 & -0.68 & -0.66 \\
\hline 5 & PA 801 x DWDa 1402 & 0.88 & 1.02 & 0.81 & $0.90^{*}$ & 0.37 & 0.36 & 1.20 & 0.64 \\
\hline 6 & PA 801 x JLA 794 & 0.01 & 0.01 & 0.14 & 0.05 & -1.58 & -1.07 & -1.46 & $-1.37 * *$ \\
\hline 7 & PA 801 x Digvijay & 0.58 & 0.69 & 0.94 & $0.73 *$ & 0.28 & 0.29 & -0.13 & 0.14 \\
\hline 8 & PA 801 x G.Cot 23 & -0.30 & -0.11 & -0.21 & -0.20 & 0.08 & 0.70 & -0.06 & 0.24 \\
\hline 9 & PA $740 \times$ AKA 8 & 0.89 & 0.74 & 0.94 & $0.85^{*}$ & 0.40 & 0.80 & 1.46 & 0.88 \\
\hline 10 & PA 740 x PhuleDhanwantary & -0.03 & 0.21 & 0.08 & 0.08 & 0.32 & 0.027 & -0.67 & -0.10 \\
\hline
\end{tabular}


Table.2 (Contd...)

\begin{tabular}{|c|c|c|c|c|c|c|c|c|c|}
\hline \multirow{2}{*}{$\begin{array}{l}\text { Sr. } \\
\text { No. }\end{array}$} & \multirow[t]{2}{*}{ Genotype } & \multicolumn{4}{|c|}{ Fibre strength (g/tex) } & \multicolumn{4}{|c|}{ Ginning percentage $(\%)$} \\
\hline & & $\mathbf{E}_{1}$ & $\mathbf{E}_{2}$ & $\mathbf{E}_{3}$ & Pooled & $\mathbf{E}_{1}$ & $\mathbf{E}_{2}$ & $\mathbf{E}_{3}$ & Pooled \\
\hline 11 & PA $740 \times$ CNA 449 & -0.057 & -0.01 & 0.12 & 0.07 & 0.07 & 0.15 & -0.13 & 0.033 \\
\hline 12 & PA $740 \times$ HD 514 & $2.55^{* *}$ & 2.61 & $2.64 * *$ & $2.60 * *$ & 0.41 & 0.43 & 0.56 & 0.46 \\
\hline 13 & PA $740 \times$ DWDa 1402 & $-2.08 * *$ & $-2.09 * *$ & $-2.13 * *$ & $-2.10 * *$ & 0.007 & -0.11 & -0.56 & -0.22 \\
\hline 14 & PA $740 \times$ JLA 794 & -0.83 & -0.83 & -0.91 & $-0.85 *$ & -1.10 & -1.16 & -1.28 & $-1.18 *$ \\
\hline 15 & PA 740 x Digvijay & 0.12 & -0.01 & -0.05 & 0.022 & 0.028 & 1.25 & 1.28 & 0.85 \\
\hline 16 & PA 740 x G.Cot 23 & -0.55 & -0.62 & -0.69 & -0.62 & -0.15 & -1.39 & -0.64 & -0.72 \\
\hline 17 & PA $812 \times$ AKA 8 & 0.84 & 0.95 & 1.01 & $0.93 *$ & 0.13 & 0.54 & 0.34 & 0.33 \\
\hline 18 & PA 812 x PhuleDhanwantary & 0.31 & 0.28 & 0.23 & 0.27 & 0.33 & 0.36 & 0.73 & 0.47 \\
\hline 19 & PA 812 x CNA 449 & $1.93 * *$ & $2.07 * *$ & $2.13 * *$ & $2.04 * *$ & -0.21 & -0.28 & -0.59 & -0.36 \\
\hline 20 & PA 812 x HD 514 & 0.18 & 0.09 & -0.03 & 0.08 & -1.49 & -1.15 & -1.07 & $-1.23 *$ \\
\hline 21 & PA 812 x DWDa 1402 & $-1.50 *$ & $-1.56 * *$ & $-1.58 *$ & $-1.55^{* *}$ & -0.28 & -0.68 & -0.82 & -0.59 \\
\hline 22 & PA 812 x JLA 794 & -0.53 & -0.52 & -0.61 & -0.55 & 0.73 & 0.91 & 0.70 & 0.78 \\
\hline 23 & PA 812 x Digvijay & -0.82 & -0.90 & -0.88 & $-0.86^{*}$ & 0.71 & 0.60 & 0.79 & 0.70 \\
\hline 24 & PA 812 x G.Cot 23 & -0.41 & -0.41 & -0.28 & -0.36 & 0.08 & -0.32 & -0.08 & -0.10 \\
\hline 25 & PA 809 x AKA 8 & $1.22 *$ & $1.22 *$ & 1.09 & $1.17 * *$ & -0.81 & -0.56 & 0.11 & -0.42 \\
\hline 26 & PA 809 x PhuleDhanwantary & $3.27 * *$ & 3.38 & $3.53 * *$ & $3.39 * *$ & -1.05 & -0.27 & -0.01 & -0.43 \\
\hline 27 & PA 809 x CNA 449 & $-1.57 *$ & $-1.71 * *$ & $-1.77 * *$ & $-1.68 * *$ & 0.14 & -0.75 & -0.55 & -0.38 \\
\hline 28 & PA 809 x HD 514 & -0.83 & -0.70 & -0.86 & $-0.79 *$ & -0.54 & -0.98 & -0.30 & -0.60 \\
\hline 29 & PA 809 x DWDa 1402 & 0.67 & 0.69 & 0.73 & 0.69 & 0.43 & $1.66^{*}$ & -0.06 & 0.67 \\
\hline 30 & PA 809 x JLA 794 & 1.13 & 0.94 & $1.28 *$ & $1.11 * *$ & $1.86^{*}$ & 1.45 & 1.59 & $1.63 * *$ \\
\hline 31 & PA 809 x Digvijay & $-1.96 * *$ & $-1.77 * *$ & $-1.91 * *$ & $-1.88 * *$ & -0.23 & -0.18 & -1.01 & -0.47 \\
\hline 32 & PA 809 x G.Cot 23 & $-1.94 * *$ & -2.05 & $-2.08 * *$ & $-2.02 * *$ & 0.20 & -0.36 & 0.24 & 0.025 \\
\hline 33 & PA $785 \times$ AKA 8 & -0.48 & -0.47 & -0.50 & -0.48 & 1.47 & 1.55 & 0.87 & $1.29 * *$ \\
\hline 34 & PA 785 x PhuleDhanwantary & -0.32 & -0.86 & -0.76 & -0.64 & 0.29 & -0.37 & -0.60 & -0.22 \\
\hline 35 & PA 785 x CNA 449 & 0.41 & 0.30 & 0.18 & 0.29 & 1.04 & 1.30 & $3.01 * *$ & $1.78^{* *}$ \\
\hline 36 & PA $785 \times$ HD 514 & -0.79 & -0.59 & -0.26 & -0.55 & -0.85 & 0.46 & -0.23 & -0.20 \\
\hline 37 & PA 785 x DWDa 1402 & -0.41 & -0.43 & -0.45 & -0.43 & $-2.01 *$ & $-2.41 * *$ & $-2.29 *$ & $-2.23 * *$ \\
\hline 38 & PA 785 x JLA 794 & $-1.55^{*}$ & -1.44 & $-1.59 *$ & $-1.52 * *$ & -0.48 & -0.24 & -1.04 & -0.58 \\
\hline 39 & PA 785 x Digvijay & $1.87 * *$ & $2.09 * *$ & $1.94 * *$ & $1.96^{* *}$ & -0.39 & -1.08 & 0.13 & -0.44 \\
\hline 40 & PA 785 x G.Cot 23 & $1.27 *$ & 1.39 & $1.45^{*}$ & $1.37 * *$ & 0.92 & 0.78 & 0.14 & 0.61 \\
\hline
\end{tabular}


Table.2 (Contd...)

\begin{tabular}{|c|c|c|c|c|c|c|c|c|c|}
\hline \multirow{2}{*}{$\begin{array}{l}\text { Sr. } \\
\text { No. }\end{array}$} & \multirow[t]{2}{*}{ Genotype } & \multicolumn{4}{|c|}{ Fibre strength (g/tex) } & \multicolumn{4}{|c|}{ Ginning percentage $(\%)$} \\
\hline & & $\mathbf{E}_{1}$ & $\mathbf{E}_{2}$ & $\mathbf{E}_{3}$ & Pooled & $\mathbf{E}_{1}$ & $\mathbf{E}_{2}$ & $\mathbf{E}_{3}$ & Pooled \\
\hline 41 & PA $832 \times$ AKA 8 & $-1.68 * *$ & $-1.43 * *$ & $-1.54 * *$ & $-1.54 * *$ & -0.07 & -0.89 & -0.98 & -0.65 \\
\hline 42 & PA 832 x PhuleDhanwantary & -0.72 & -0.70 & -0.68 & -0.68 & -0.10 & -0.05 & -0.78 & -0.31 \\
\hline 43 & PA 832 x CNA 449 & $-1.30 *$ & $-1.33 *$ & $-1.33 * *$ & $-1.33 * *$ & -1.12 & -1.11 & -1.02 & $-1.08 *$ \\
\hline 44 & PA $832 \times$ HD 514 & 1.05 & 0.96 & $0.96 * *$ & $0.96 * *$ & 0.49 & -0.10 & 0.34 & 0.24 \\
\hline 45 & PA 832 x DWDa 1402 & -0.30 & -0.29 & -0.30 & -0.30 & 0.18 & -0.07 & 0.39 & 0.16 \\
\hline 46 & PA 832 x JLA 794 & 0.32 & 0.28 & 0.31 & 0.31 & -1.32 & -0.59 & -0.28 & -0.72 \\
\hline 47 & PA 832 x Digvijay & $1.76 * *$ & $1.68 * *$ & $1.72 * *$ & $1.72 * *$ & 1.38 & 1.14 & 1.01 & $1.17 *$ \\
\hline 48 & PA 832 x G.Cot 23 & 0.87 & 0.83 & $0.85 *$ & $0.85^{*}$ & 0.56 & $1.69 *$ & 1.33 & $1.19 *$ \\
\hline 49 & PAIG $346 \times$ AKA 8 & $-1.40 *$ & $-1.38 *$ & $-1.36 * *$ & $-1.36^{* *}$ & $-1.69^{*}$ & -1.49 & $-1.99 *$ & $-1.72 * *$ \\
\hline 50 & PAIG 346 xPhuleDhanwantary & -0.09 & -0.01 & -0.07 & -0.07 & 0.44 & 0.49 & 0.34 & 0.42 \\
\hline 51 & PAIG 346 x CNA 449 & -0.70 & -0.58 & -0.65 & -0.65 & -1.01 & 0.05 & -0.66 & -0.53 \\
\hline 52 & PAIG 346 x HD 514 & $-1.51 *$ & -1.44 & $-1.43 * *$ & $-1.43 * *$ & $2.52 * *$ & $2.10^{*}$ & 1.40 & $2.00 * *$ \\
\hline 53 & PAIG 346 x DWDa 1402 & $2.75^{* *}$ & $2.66^{* *}$ & $2.78 * *$ & $2.78 * *$ & 1.31 & 1.25 & $2.14 *$ & $1.56^{* *}$ \\
\hline 54 & PAIG 346 x JLA 794 & $1.46^{*}$ & 1.56 & $1.45 * *$ & $1.45^{* *}$ & $1.89 *$ & 0.71 & $1.76^{*}$ & $1.45^{* *}$ \\
\hline 55 & PAIG 346 x Digvijay & $-1.56^{*}$ & $-1.78 * *$ & $-1.70 * *$ & $-1.70 * *$ & $-1.78 *$ & $-2.01 *$ & $-2.07 *$ & $-1.95 * *$ \\
\hline \multirow[t]{5}{*}{56} & PAIG 346 x G.Cot 23 & 1.06 & 0.97 & $0.99 * *$ & $0.99 * *$ & $-1.69 *$ & -1.10 & -0.92 & $-1.23 *$ \\
\hline & S.E. \pm & & & & & & & & \\
\hline & Lines & 0.213 & 0.184 & 0.13 & 0.13 & 0.287 & 0.285 & 0.309 & 0.17 \\
\hline & Tester & 0.228 & 0.197 & 0.14 & 0.14 & 0.307 & 0.305 & 0.330 & 0.18 \\
\hline & Crosses & 0.604 & 0.521 & 0.32 & 0.32 & 0.813 & 0.807 & 0.874 & 0.48 \\
\hline
\end{tabular}

*** - Significant at 5 per cent and 1 per cent level, respectively 
Among testers, highest positive significant GCA was observed in DWDa 1402 (1.92). It showed relatively closer GCA across the environments $\left(\mathrm{E}_{1}=1.93, \mathrm{E}_{2}=1.93, \mathrm{E}_{3}=1.92\right)$. It was followed by CNA 449 (0.98) and JLA $794(0.85)$.

Among the crosses, highest positive significant SCA was reported in PA $809 \mathrm{x}$ PhuleDhanwantary (3.39). It showed high positive significant $\mathrm{SCA}$ at $\mathrm{E}_{3}$ (3.53) than $\mathrm{E}_{2}$ (3.38) and $E_{1}$ (3.27). Next highest SCA was observed in PAIG 346 x DWDa 1402 (2.78) which also exhibited higher SCA at $\mathrm{E}_{3}(2.94)$ than $E_{1}$ (2.75) and $E_{2}$ (2.66). Another cross with significantly positive SCA was PA $740 \mathrm{x}$ HD 514 (2.60). It showed higher SCA at $\mathrm{E}_{3}$ (2.64) than $E_{2}$ (2.61) and $E_{1}$ (2.55). Among the interspecific group, out of 14 crosses, six crosses recorded significantly positive SCA in pooled analysis. Cross PA 785 x Digvijay (1.96), PA 832 x Digvijay (1.72) and PA 785 x G.Cot 23 (1.37) exhibited highly significant and positive SCA effects for this trait in all the locations as well as in pooled analysis.

\section{Ginning percentage (\%)}

Among lines highest positively significant GCA for increasing ginning percentage was observed in PA 809 (0.55). It exhibited highest GCA at $\mathrm{E}_{1}(0.76)$ than $\mathrm{E}_{2}(0.54)$ and $\mathrm{E}_{3}$ (0.36). Second highest GCA among lines was observed in PA 740 (0.43). It showed significantly higher GCA at $\mathrm{E}_{1}(0.60)$ and $\mathrm{E}_{2}$ (0.57) than $\mathrm{E}_{3}$ (0.13). Among the testers highest positive significant GCA was imparted by HD 514 (1.67). It showed relatively closer GCA across the environments $\left(\mathrm{E}_{1}=1.77, \mathrm{E}_{2}=1.74, \mathrm{E}_{3}=\right.$ 1.50). The combination of the two best general combiners i.e. PA 809 x HD 514 possessed negative non-significant SCA for ginning percentage.

The best combination for significantly positive highest SCA was PAIG 346 x HD
514 (2.00). It showed significantly highest SCA at $\mathrm{E}_{3}$ (2.14) than $\mathrm{E}_{1}$ (1.31) and $\mathrm{E}_{2}$ (1.25). Next significantly highest SCA was observed in PA $785 \times$ CNA 449 (1.78). It possessed significantly higher SCA at $\mathrm{E}_{3}$ (3.01) than $\mathrm{E}_{2}$ (1.30) and $\mathrm{E}_{1}$ (1.04). Among the interspecific crosses, only two crosses viz., PA $832 \mathrm{x}$ G.Cot 23 (1.19) and PA 832 x Digvijay (1.17) recorded significant and positive SCA effects in pooled analysis.

For fibre quality parameters, PA 740 X HD 514 was found to be the best combination exhibiting significant SCA for UHML (1.40), micronaire (-0.21), uniformity index (0.19) and fibre strength (2.60) pooled over the locations. Selection in further generations would be rewarding to get best transgressive segregants for fibre quality.

The estimates of GCA effect further revealed that the parental lines exhibited high to average GCA effect for one or more fibre quality characters. Selection of the most encouraging cross combination needs high SCA along with high GCA effects of respective parents. Almost identical results have been reported by Tomar and Singh (1996), Saxena et al., (1998), Kajjidoni et al., (2002), Deshpande et al., (2003), Maisuria et al., (2006), Preetha and Ravindran (2008), Laxman (2010), Kumar et al., (2014), Madhuri et al., (2014), Patel and Choudhari (2015) and Shakeel et al., (2016). Parents possessing good gca for yield and yield contributing characters need to be widely used in breeding programme for combining desirable characters.

\section{References}

Comstock, R.E. and Robinson, H.F. 1952. Estimation of average dominance of genes. In " Heterosis " Ed. G.W.Gowen. IowaState College Press, Ames, Iowa.494-516.

Deshpande, L.A., Baig, K.S. and Nagare, Y.P. 
2003.Combining ability studies for yield, fibre quality and morphological traits in desi cotton (G. arboreum L.). J. Maharashtra Agric. Univ., 28 (1): 3639.

Kajjidoni, S.T., Patil, S.J., Salimath, P.M. and Khadi, B.M. 2002.Combining ability in GMS based and conventional crosses of G. arboreum. L. ISCI Journal, 27 (2): 84-88.

Kempthorne, O. 1957. "An Introduction to Genetic Statistics" JohnWiley and Sons Inc., New York, pp. 453-471.

Kumar K.S., AshokkumarK., andRavikesavan R. 2014. Genetic effects for combining ability studies for yield and fibre quality traits in diallel crosses of upland cotton (Gossypium hirsutum L.) Academic Journals.13 (1), pp. 119-126.

Laxman, S. 2010. Diallel analysis for combining ability for seed cotton yield and its components in desi cotton (G.arboreum L.). J. Cotton Res. Dev., 24(1): 26-28.

Madhuri S., Anita, S., Mashal, G.S. and Deshmukh S.B. 2014. Combining ability and heterosis for seed cotton yield, its components and quality in Gossypium hirsutum L. Indian J. Agric. Res., 49(2): 154-159.

Maisuria, A.T., Patel, J.C., Patel, K.G. and Solanki, B.G. 2006. Study of heterosis and combining ability in GMS based Asiatic cotton (G. herbaceum L.). ISCI Journal, 88-92.

Patel N.N., and Choudhari Pinal .2015.Combining ability study for yield and its component traits through line $x$ tester mating design in Asiatic (Gossypium herbaceum L.) cotton. J. Cotton Res. Dev. 29 (1):19-22.

Preetha, S. and Raveendran, T.S. 2008.Combining ability and heterosis for yield and fibre quality traits in line $\mathrm{x}$ tester crosses of upland cotton (Gossypium hirsutum L.). Int. J. Pl. Breed. Genet., 2:64-74.

Saxena, U., Saxena, M.K., Acharya, V.N. and Ray, N. 1998.Combining ability analysis for seed cotton yield and other characters in $G$. arboreum L. ISCI Journal, 23 (2) : 137-139.

Shakeel, A. Saeed, W. Nazeer, Z. U. Zia and Malik W. 2016. Combining ability analysis and heterotic studies for within -boll yield components and fibre quality in cotton. The Journal of Animal \& Plant Sciences, 26 (1):156-162.

Tomar, S.K. and Singh, S.P. 1996. Genetics of yield and yield component over location indesi cotton $(G$. arboreum L.). Indian J. Genet., 56(1): 89 - 93.

\section{How to cite this article:}

Chinchane, V. N., D. B. Deosarkar, K. S. Baig and Kalpande, H. V. 2020. Combining Ability Analysis for Fibre Quality Traits in Desi Cotton (Gossypium arboreum L.) Across the Environments. Int.J.Curr.Microbiol.App.Sci. 9(01): 1885-1896.

doi: https://doi.org/10.20546/ijcmas.2020.901.211 